\title{
Evaluation of a Portable Microchip Electrophoresis Fluorescence Detection System for the Analysis of Amino Acid Neurotransmitters in Brain Dialysis Samples
}

\author{
Nathan J. Oborny, ${ }^{* 1, * 2}$ Elton E. Melo Costa, ${ }^{* 2, * 3}$ Leena Suntornsuk, ${ }^{* 2, * 4}$ Fabiane C. Abreu, ${ }^{* 3}$ \\ and Susan M. LUNTE*1,*2,*5† \\ *1 Department of Bioengineering, University of Kansas, Lawrence, KS 66045, USA \\ *2 Ralph N. Adams Institute for Bioanalytical Chemistry, University of Kansas, Lawrence, KS 66047, USA \\ *3 Institute of Chemistry and Biotechnology, Federal University of Alagoas, Alagoas, Brazil \\ *4 Department of Pharmaceutical Chemistry, Faculty of Pharmacy, Mahidol University, Bangkok, Thailand \\ *5 Department of Chemistry, University of Kansas, Lawrence, KS 66045, USA
}

\begin{abstract}
A portable fluorescence detection system for use with microchip electrophoresis was developed and compared to a benchtop system. Using this system, six neuroactive amines commonly found in brain dialysate (arginine, citrulline, taurine, histamine, glutamate, and aspartate) were derivatized offline with naphthalene-2,3-dicarboxaldehyde/cyanide, separated electrophoretically, and detected by fluorescence. The limits of detection for the analytes of interest were $50-250 \mathrm{nM}$ for the benchtop system and $250 \mathrm{nM}-1.3 \mu \mathrm{M}$ for the portable system, both of which were adequate for most analyte detection in brain microdialysis samples. The portable system was then demonstrated for the detection of the same six amines in a rat brain microdialysis sample.
\end{abstract}

Keywords Microchip electrophoresis, amino acid neurotransmitter, microdialysis, fluorescence detection, naphthalene2,3-dicarboxaldehyde, neuroactive amine

(Received October 21, 2015; Accepted November 9, 2015; Published January 10, 2016)

\section{Introduction}

Microdialysis (MD) is a popular in vivo sampling method that has been used to monitor neurotransmitters in both animal models and humans. In particular, microdialysis sampling has been employed to monitor chemicals in the brains of traumatic brain injury patients in intensive-care units (ICU). MD sampling can also be used to monitor the extent of tissue damage and the efficacy of treatment simultaneously through continuous and long-term sampling of multiple biomarkers. ${ }^{1-3}$

The process of MD sampling has been described elsewhere extensively. ${ }^{4-6}$ Of relevance here, however, is the fact that the recovery of any analyte of interest is governed by the flow rate of the perfusate liquid. ${ }^{7}$ At a flow rate of $1 \mu \mathrm{L} / \mathrm{min}$, typical analyte recoveries for small molecules are in the range of 10 $40 \%$. Lower flow rates result in higher analyte recoveries, approaching $100 \%$ at $100 \mathrm{~nL} / \mathrm{min}$, but with smaller volumes of dialysate collected per unit time. ${ }^{8,9}$ This tradeoff dictates which analytical technique can be used and how often analysis can be performed.

Typically, MD samples are collected until sufficient volumes are acquired to allow the use of the desired analytical method with requisite sensitivity and selectivity. The most common method employed for the analysis of microdialysis samples is

$\dagger$ To whom correspondence should be addressed.

E-mail: slunte@ku.edu liquid chromatography (LC) coupled to electrochemical, fluorescence, mass spectrometry (MS), or absorbance detection. ${ }^{10}$ However, the time required to obtain a dialysate sample large enough for many LC-based assays can reduce the temporal resolution of the technique as well as add a significant delay between the sample acquisition and clinical response. In order to address a clinical need for an analytical technique that can continuously analyze small volume samples, and selectively detect very low analyte concentrations with good temporal resolution, several groups have developed on-line MD methods using capillary (CE) and microchip electrophoresis (ME). ${ }^{11,12}$ These systems, when combined with a sensitive detection method, such as laser- (or light emitting diode-) induced fluorescence (ME-LIF and ME-LEDIF), can rapidly analyze nanoliter-volume samples in a continuous manner, while providing clinicians with near real-time data.

There are many examples concerning the use of CE to monitor amino acid neurotransmitters in microdialysis samples, ${ }^{13-18}$ and other examples have been reviewed by Poinsot et al. ${ }^{19}$ However, these systems typically use off-line sample collection and precolumn derivatization, requiring samples large enough to be handled accurately off-line. Coupling CE or ME directly to MD makes it possible for much smaller sample volumes to be analyzed, leading to better temporal resolution as long as the method displays the requisite sensitivity. ${ }^{20}$ To this end, our group previously developed a polydimethylsiloxane (PDMS)based microchip electrophoresis device that was coupled to microdialysis (MD-ME) for the continuous on-line monitoring 
of amino acid neurotransmitters. ${ }^{17,18}$ The microchip was completely integrated with the MD sampling system, which provided on-line derivatization of the amino acids with naphthalene-2,3-dicarboxyaldehyde/cyanide $\left(\mathrm{NDA} / \mathrm{CN}^{-}\right)$, a flow-gate interface for injection, and electrophoresis separation using a serpentine channel with LIF detection. Although the chip itself was small, the associated instrumentation needed for fluorescence detection was quite large, and thus not amenable to a clinical setting.

The aim of the present work is to expand on the theme of miniaturization and integration of the on-line MD-ME-LIF device with the goal to obtain a system that can be used for the near real-time monitoring of amine-based neurotransmitters in the ICU or research laboratory. The particular focus of this report is miniaturization of the fluorescence detection system. To evaluate the system, figures of merit for the separation and detection of several NDA/CN-derivatized amines commonly found in rat brain dialysate were determined using a conventional benchtop ME-LIF system employing an epifluoresence microscope. These results were then compared to those obtained using the newly developed portable ME-LEDIF system. The portable system described here could potentially be placed near patients in an ICU (or next to animals for animal studies), permitting continuous near real-time monitoring of these neuroactive amines in injured brain tissue to assess damage and to monitor treatment.

\section{Experimental}

\section{Reagents and chemicals}

Arginine (Arg), aspartate (Asp), citrulline (Cit), glutamate (Glu), histamine (Hist), and taurine (Tau) were obtained from Sigma Aldrich (St. Louis, MO). Standards of each amine were prepared at $2 \mathrm{mM}$ concentrations in $18.2 \mathrm{M} \Omega / \mathrm{cm}$ deionized water (Millipore, Billerica, MA). Subsequent dilutions of each stock solution were made prior to analysis. Naphthalene-2,3dicarboxaldehyde (NDA) (Invitrogen, Carlsbad, CA) was prepared in acetonitrile (Fisher Scientific, Pittsburgh, PA) to a concentration of $5 \mathrm{mM}$. Sodium cyanide $(\mathrm{NaCN})$ (Sigma Aldrich) was dissolved in water to a final concentration of $10 \mathrm{mM}$. Stock solutions of both NDA and $\mathrm{NaCN}$ were made weekly and stored at $4^{\circ} \mathrm{C}$, while being protected from light exposure. Stock solutions of sulfobutylether- $\beta$-cyclodextrin (SBEC) (Life Technologies, Grand Island, NY) were made on a weekly basis to a concentration of $10 \mathrm{mM}$ in deionized water, and stored at $4^{\circ} \mathrm{C}$. The background electrolyte (BGE) consisted of $1.4 \mathrm{mM}$ SBEC, $10 \%$ by volume HPLC-grade dimethylsulfoxide (Fisher Scientific), and sodium tetraborate (Sigma Aldrich) at a final concentration of $15 \mathrm{mM}$. Finally, the $\mathrm{pH}$ of the BGE was measured using a $\mathrm{pH}$ meter and adjusted to 9.2 with $1 \mathrm{M}$ sodium hydroxide (Fisher Scientific).

\section{Animal surgery and microdialysis sampling}

Male Sprague-Dawley rats were housed in temperaturecontrolled rooms with free access to food and water prior to surgery. Rats were fully anesthetized prior to surgery through inhalation of isofluorane, followed by the injection of a mixture of ketamine $(67.5 \mathrm{mg} / \mathrm{kg})$, xylazine $(3.4 \mathrm{mg} / \mathrm{kg})$, and acepromazine $(0.67 \mathrm{mg} / \mathrm{kg})$. To maintain anesthesia throughout the surgery, doses of ketamine were administered by intramuscular injections. The body temperature was maintained using a Homeothermic Blanket Control unit (Harvard Apparatus, Holliston, MA) set at $37^{\circ} \mathrm{C}$, and rats were given saline doses to keep them hydrated. All surgical instruments were sterilized

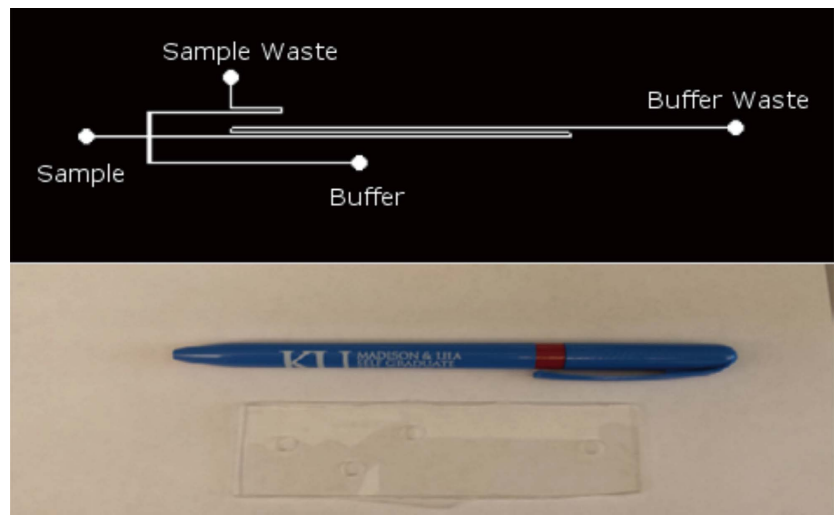

Fig. 1 Top: $15 \mathrm{~cm}$-length serpentine chip design with $2.7 \mathrm{~cm}$-length side channels for sample waste and buffer, $0.5 \mathrm{~cm}$ length for sample inlet. All channels were $15 \mu \mathrm{m}$ deep by $70 \mu \mathrm{m}$ wide. Bottom: Actual glass chip used in these studies.

before usage and after the surgical procedures. A guide cannula followed by a microdialysis probe with a 4-mm membrane was inserted in the striatum region at stereotaxic coordinates $\mathrm{A} / \mathrm{P}$ $+0.7, \mathrm{M} / \mathrm{L}-2.7, \mathrm{~V} / \mathrm{D}-3.4 .^{17,21}$ Microdialysis samples were collected by perfusing the probe (CMA, North Chelmsford, MA) with an artificial spinal fluid (aCSF) at $1 \mu \mathrm{L} / \mathrm{min}$. Animal experiments were performed in accordance with regulations of the Institutional Animal Care and Use Committee (IACUC) at the University of Kansas, which operates under accreditation from the Association for Assessment and Accreditation of Laboratory Animal Care (AAALAC).

\section{Derivatization reaction}

Derivatization of the amino acid standards and MD sample was carried out using equal parts by volume of $5 \mathrm{mM}$ NDA and $10 \mathrm{mM} \mathrm{NaCN}, 15 \mathrm{mM}$ boric buffer (pH 9.2), and sample. NDA reacts with primary amines in the presence of cyanide to produce fluorescent 1-cyanobenz $[f]$ isoindole $(\mathrm{CBI})$ products.

\section{Microchip electrophoresis (ME)}

The glass microfluidic devices used in these studies were fabricated using standard photolithographic techniques, as reported previously. ${ }^{22,23}$ To separate the six target analytes, a $15-\mathrm{cm}$ serpentine separation channel with $3-\mathrm{cm}$ side channels (Fig. 1) was used. All channels were $15 \mu \mathrm{m}$ deep and approximately $70 \mu \mathrm{m}$ wide. Before each use, the chip was conditioned with $0.1 \mathrm{M} \mathrm{HCl}$, water, $0.1 \mathrm{M} \mathrm{NaOH}$, and then water again. Each solution was passed through the microchannels for $10 \mathrm{~min}$ via the application of negative pressure to one of the ports. Finally, the channels were filled with BGE using the same negative pressure procedure prior to use.

An UltraVolt HV Rack high-voltage power supply (Ronkonkoma, NY) was employed for the electrophoresis experiments and controlled by software written in Labview (National Instruments, Austin, TX). In these experiments, a voltage of $10 \mathrm{kV}$ was applied at the buffer reservoir and $7 \mathrm{kV}$ at the sample reservoir for an overall separation field strength of $420 \mathrm{~V} / \mathrm{cm}$. For sample electrokinetic injection, the injection time was $0.7 \mathrm{~s}$ and the analysis time lasted $420 \mathrm{~s}$.

\section{Benchtop ME-LIF system}

Fluorescence was chosen as the detection method in these studies because it provides high sensitivity and, in the case of $\mathrm{NDA} / \mathrm{CN}$ derivatization, selectivity for primary amines. ${ }^{24}$ 
(a)

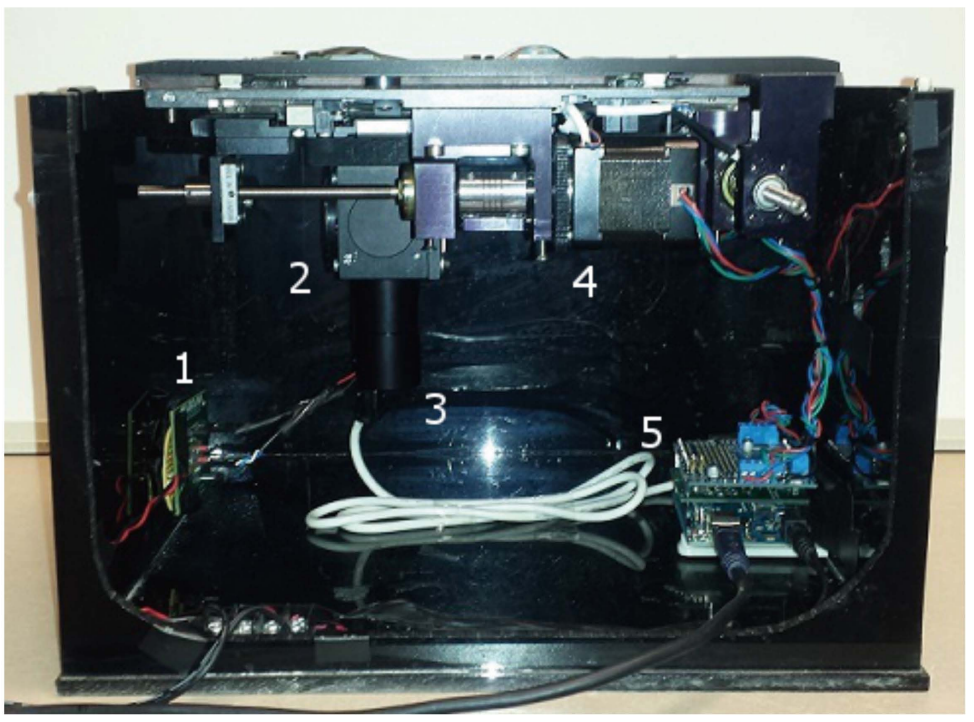

(b)

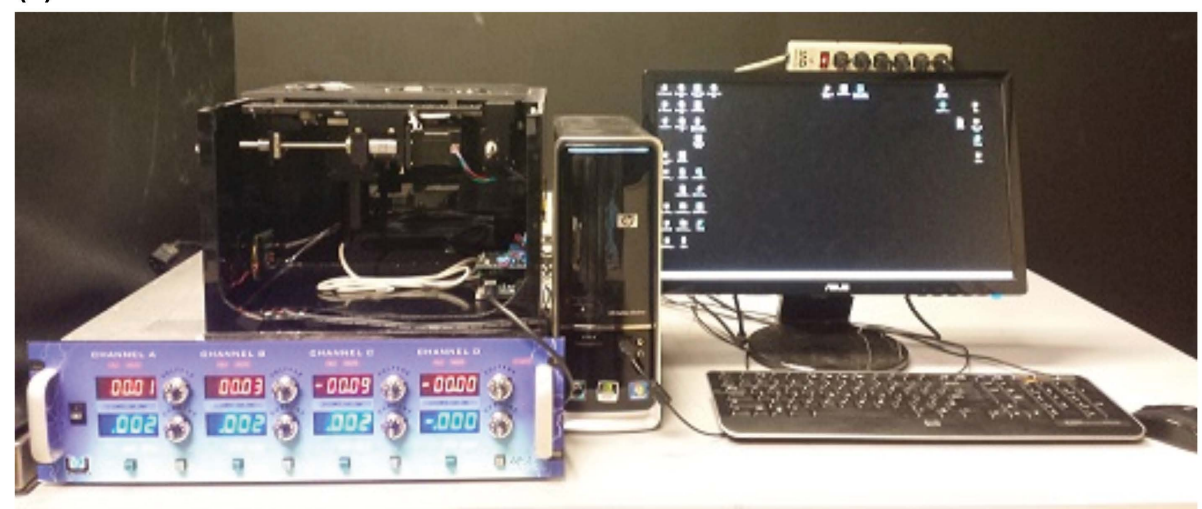

Fig. 2 (a) Close-up view of the portable system. 1, LED current driver board; 2, optical mounting containing dichroic mirror, LED, objective lens, and optical filter; 3, photodiode and preamplifier board; 4, positioning motors, seen here from the side; 5 , arduino microcontroller with stacked motor driver shield and amplifier and analog to digital converter shield connected to preamplifier via white USB cable. The system itself measures $12^{\prime \prime}$ wide $\times 11^{\prime \prime}$ deep $\times 8^{\prime \prime}$ tall and weighs approximately $10 \mathrm{lbs}$. (b) Portable system shown with Ultravolt high voltage supply and PC for scale.

The cyanobenz $(f)$ isoindole products of the NDA/CN reaction exhibit two excitation maxima in the visible range at approximately 420 and $442 \mathrm{~nm} \cdot{ }^{25,26}$ For the benchtop ME system used in these studies, excitation was accomplished using either a 445-nm PhoxX diode laser (Market Tech, Scotts Valley, CA) or a 442-nm CL-2000 diode laser (Crystal Laser, Reno, NV). The excitation light source was coupled to an epifluorescence microscope (Nikon, Melville, NY) via a fiber optic cable; it was optically filtered using a 445-nm band pass filter, and was focused on the separation channel approximately $0.2 \mathrm{~cm}$ from the waste reservoir via a long-pass dichroic mirror that directed the light to the chip and a $40 \times$ objective lens to focus onto the channel. The emission maxima for the products of the NDA/CN reaction occur at $490 \mathrm{~nm} .{ }^{25}$ Once again, the epifluorescence microscope was used to focus the emission light through the long-pass dichroic mirror and long-pass edge filter (480 $\mathrm{nm}$ cutoff) before it was focused onto a photomultiplier tube (PMT). Signal acquisition from the PMT was performed using a National Instruments NI USB-6229 data acquisition card and Labview software, following amplification and low pass filtering with a $3-\mathrm{Hz}$ filter cutoff using a Model SR570 amplifier from Stanford Research Systems (Sunnyvale, CA). Origin software Ver. 8.2 (Origin Lab Corp., Northampton, MA) was used to analyze the subsequently collected data.

\section{Portable ME-LEDIF system}

The portable system has many commonalities with the benchtop ME system but also has several differences. As in the benchtop ME, fluorescence detection was accomplished by filtering and focusing excitation light onto the separation channel, followed by filtering and focusing subsequently emitted light onto a detector. However, in the case of the portable system, a 420-nm LED (LED Engin, San Jose, CA) was used instead of a laser. The LED was powered using a custom current driver circuit and constructed using a frequency-controlled variable current driver, a MAX16836 (Maxim Integrated), driven by an LM555 timer IC (Texas Instruments, Dallas, TX). It was found that an input signal of $1.6 \mathrm{kHz}$ resulted in a stable current output of $250 \mathrm{~mA}$.

A microscope stage purchased from eBay is shown mounted in Fig. 2. This stage, which was originally designed to be motor driven, was modified using 3D-printed motor brackets to couple 
two NEMA 17 stepper motors to the stage. This allowed controlled movement in the $\mathrm{X}$ and $\mathrm{Y}$ directions as the optics mount moved along threaded rods while fixing the $\mathrm{Z}$ direction, such that the channel was at the focal point of a $100 \times$ objective. These motors were subsequently driven by a stepper motor driver shield for the Arduino microcontroller or, alternatively, could be manually adjusted.

As mentioned previously, an LED was employed as the excitation light source in the portable system and was mounted parallel to the chip. This LED was collimated using a planoconvex lens, followed by spatial focusing via a 1-mm pinhole. The light from the LED was directed upward through an objective lens via a long-pass dichroic mirror with a 470-nm cutoff (Thorlabs, Newton, NJ) and mounted at a $90^{\circ}$ angle. Emission light was focused via the same objective lens through the long-pass dichroic mirror followed by a long-pass emission filter and was finally focused onto a detection point using a second plano-convex lens, Model LA1951-A-N-BK7 (Thorlabs). The final long-pass emission filter also had a cutoff of $470 \mathrm{~nm}$ (Thorlabs), allowing light of any longer wavelength to pass on to the detector.

The detector itself consisted of an OPT301 combination transimpedance amplifier/silicon photodiode (Texas Instruments) in a custom circuit, mounted on a $1^{\prime \prime}$-dia printed circuit board (PCB). This PCB was subsequently mounted in the optics holder, such that the OPT301 was centered at the focal point of the plano-convex lens. This PCB was connected to the secondary amplifier via a 4-pin shielded USB cable, which also supplied the OPT301 with power. Finally, the output of the OPT301 was amplified by a custom second-stage amplifier circuit using an OPA726 IC (Texas Instruments) before passing the amplified signal to an analog-to-digital (ADC) converter, a Max11210 IC (Maxim Integrated, San Jose, CA). The secondary amplification and ADC circuit was designed in the format of an Arduino shield, which allowed it to be easily interfaced with the open source Arduino Uno Microcontroller used for signal processing as well as communication to a nearby PC via USB. While future versions of the portable system will have an integrated high-voltage power supply for the separation as well as electrokinetic gates, the system used for this study relied on the UltraVolt HV Rack high-voltage power supply previously mentioned.

\section{Results and Discussion}

The on-line integration of MD to an analysis method, such as ME-LEDIF, has the potential to provide clinicians and animal researchers with minute-to-minute data regarding the tissue health of patients, or to track changes in neurotransmitters in animal models. While several groups have successfully coupled $\mathrm{MD}$ to both $\mathrm{CE}^{23}$ and $\mathrm{ME},{ }^{12,17}$ in practice, the benefits of doing so have been limited by the size of the associated equipment needed for the separation and detection. In an already crowded intensive-care unit or neurobiology laboratory, this is a significant concern, because any increased distance from the patient or animal could result in a significant delay in response due to low sample flow rates from the MD probe to the microchip. The goal of miniaturizing and creating the selfcontained ME-LIF system described here is to reduce the timelag between sampling and analysis as well as the overall size of the external equipment necessary. The aim is to create a small, portable detection system that can be placed near the patient or animal for near real-time monitoring.

To miniaturize the system as much as possible, several design decisions were made. The first of these was the choice of an LED excitation light source rather than a laser. There are three reasons for this. First, lasers are considerably more expensive than LEDs. Second, the mechanical robustness of the overall design was a consideration for portability and, typically, lasers are more fragile than LEDs. Finally, while we focused on NDA/ $\mathrm{CN}$ derivatization in this study, the easy availability of a wide range of LED wavelengths for use in future studies with other fluorogenic compounds also influenced our decision to use an LED light source.

The requirements of portability and ruggedness also affected the physical/mechanical design of the system. Because microchip sizes and designs vary from application to application, and also because it is often necessary to move the chip for cleaning purposes, the alignment of the optics to the channel needs to be moveable in this portable system, unlike those of other miniaturized systems. ${ }^{21}$ Because a high-voltage supply with wires leading to each port on the chip was necessary, moving the optics rather than the chip is the better option in order to avoid dislodging any of the high-voltage wires during alignment.

The use of the OPT301 photodiode for detection is another feature of this instrument. Other portable fluorescence detection systems $^{27,28}$ have used a more sensitive, but ultimately more complex and costly PMT for detection. In this application, however, the use of a photodiode was adequate for the LODs required in these studies, and simplified the design by eliminating the need for a high-voltage supply for the PMT.

To evaluate the portable system for determining the amines in microdialysis samples, it was necessary to compare the function of a traditional benchtop ME-LIF system to the portable ME-LEDIF system for the same set of analytes. For this purpose, a mixture of six neuroactive amines (arginine (Arg), citrulline (Cit), taurine (Tau), histamine (Hist), glutamate (Glu) and aspartate (Asp)) was derivatized using $\mathrm{NDA} / \mathrm{CN}$ and electrophoretically separated using microchip electrophoresis. For each amine, the linearity over a range of physiologically relevant concentrations (Table 1) as well as the limits of detection (LOD) and the limits of quantification (LOQ) were determined using both systems. Following this, rat brain microdialysis samples were derivatized using NDA/CN, and analyzed on both systems. For the purposes of these proof-ofconcept tests, all samples were derivatized off-line for $30 \mathrm{~min}$ prior to separation.

As can be seen in Table 1, both systems exhibited a linear response over the physiologically relevant concentration ranges. The LOD for each amine can be found in Table 1. The LODs were lower for the benchtop ME-LIF, mainly due to the use of a PMT instead of a photodiode. Five of the six amines (Arg, Cit, Tau, Glu and Asp) could be detected, and were present above the LODs determined with the portable system. The concentrations of aspartate and histamine were below the calculated LOQs and, in the case of Hist, below the LOD. A peak that co-migrated with Hist was consistently visible in the electropherogram and identified via standard Hist spiking. The presence of this peak in the MD sample implies two possibilities. The first is that a substantial increase in extracellular Hist occurred due to tissue inflammation as a result of probe insertion. ${ }^{29}$ The second is the co-migration of an as yet unknown analyte to be identified in future studies. However, even though the benchtop ME-LIF system exhibited lower LODs (and LOQs) for five of the six amines, the performance of the portable system was adequate for their detection at physiologically relevant concentrations. Figure 3 (Bottom) shows an electropherogram obtained with the portable system for a 
Table 1 Performance parameters for the benchtop and portable LIF systems

\begin{tabular}{|c|c|c|c|c|c|c|}
\hline & Arg & Cit & Tau & Hist & Glu & Asp \\
\hline Physiological range $\mathrm{e}^{4,29-31} / \mu \mathrm{M}$ & $0.6-1.5$ & $0.4-1$ & $5-20$ & $4.7-6.7 \mathrm{nM}$ & $8-22$ & $0.8-1.6$ \\
\hline \multicolumn{7}{|l|}{ Benchtop system } \\
\hline Calibration range $/ \mu \mathrm{M}$ & $0.1-10$ & $0.1-10$ & $0.1-10$ & $0.1-10$ & $0.1-10$ & $0.1-10$ \\
\hline Regression coefficient $\left(R^{2}\right)$ & 0.9956 & 0.9897 & 0.9940 & 0.9990 & 0.9792 & 0.9854 \\
\hline Limit of detection $(S / N=3) / \mu \mathrm{M}$ & 0.05 & 0.05 & 0.05 & 0.05 & 0.25 & 0.05 \\
\hline Limit of quantification $(S / N=10) / \mu \mathrm{M}$ & 0.15 & 0.15 & 0.15 & 0.15 & 1.0 & 0.15 \\
\hline Migration time, \%RSD & 1.81 & 1.46 & 1.26 & 1.34 & 1.85 & 2.61 \\
\hline Theoretical plate/m & 746485 & 693960 & 872808 & 473232 & 565178 & 600081 \\
\hline Peak resolution & 1.29 & 1.16 & 0.97 & 1.15 & 1.10 & 0.15 \\
\hline \multicolumn{7}{|l|}{ Portable system } \\
\hline Calibration range/ $\mu \mathrm{M}$ & $2.0-50$ & $2.0-50$ & $2.0-25$ & $2.0-15$ & $2.0-50$ & $2.0-50$ \\
\hline Regression coefficient $\left(R^{2}\right)$ & 0.9956 & 0.9834 & 0.9870 & 0.9977 & 0.9931 & 0.9904 \\
\hline Limit of detection $(S / N=3) / \mu \mathrm{M}$ & 0.25 & 0.36 & 0.42 & 0.37 & 1.31 & 1.21 \\
\hline Limit of quantification $(S / N=10) / \mu \mathrm{M}$ & 0.84 & 1.2 & 1.4 & 1.24 & 4.35 & 4.04 \\
\hline Migration time, \%RSD & 1.71 & 1.8 & 2.52 & 3.22 & 4.75 & 5.23 \\
\hline Theoretical plate/m & 275165 & 270431 & 331367 & 406943 & 325993 & 277798 \\
\hline Peak resolution & 2.53 & 0.96 & 1.01 & 6.09 & 2.00 & 3.08 \\
\hline
\end{tabular}
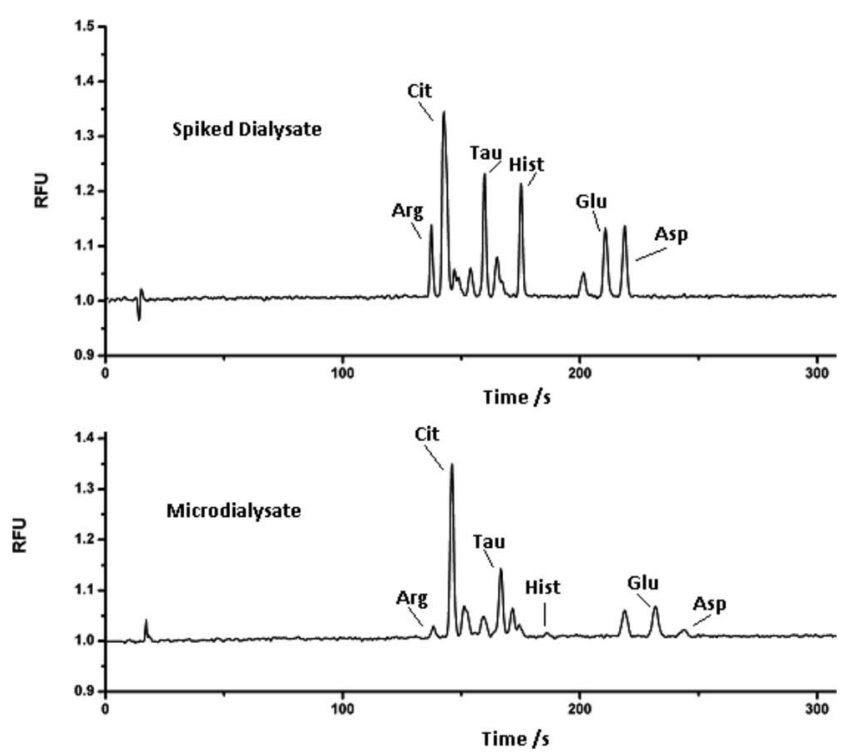

Fig. 3 (Top) MD sample spiked with an additional $5 \mu \mathrm{M}$ Arg, Cit, Tau, Hist, Glu, and Asp. (Bottom) Electropherogram of a NDA/CNderivatized microdialysate sample from striatum using the portable system.

representative microdialysis sample and (Top) the same sample spiked with $5 \mu \mathrm{M}$ (final concentration) of amine standards. In this study, identification of the peaks was based on migration time and spiking with standard solutions.

\section{Future work}

Future versions of the system will address the need for automated positioning and variable chip sizes as well as further decreases in the overall size of the system, which was larger than necessary in this design for convenience. As mentioned, the LOQs for two of the target analytes were too high for quantification in the microdialysis sample. In order to improve these LODs/LOQs, a programmable integrated circuit (PIC)-based (Microchip, Chandler, AZ) lockin-amplifier system is being developed for fluorescence detection and integrated into the system along with a high-voltage module for sample separation. The use of lower microdialysis flow rates and on-line derivatization to enhance analyte recovery and fluorescence product formation, respectively, are also being investigated. Finally, future studies will be conducted using online MD-ME sampling to look at amine changes in a rat ischemia model.

\section{Acknowledgements}

Funding for this work was provided by a Biotechnology Training Grant T32 GM-08359 and NIH COBRE P20 GM103638 as well as NIH grant R01 NS042929. Additional support was provided by the Thailand-United States Educational Foundation (TUSEF/Fulbright Thailand). We would also like to thank Dr. Rachel Saylor and Amanda Furness for the microdialysis samples and Nancy Harmony for editorial assistance.

\section{References}

1. E. Deibert and M. N. Diringer, Neurologist, 1999, 5, 313.

2. M. A. Kirkman, G. Citerio, and M. Smith, Intensive Care Med., 2014, 40, 640.

3. L. Persson and L. Hillered, J. Neurosurg., 1992, 76, 72.

4. H. Benveniste, J. Neurochem., 1989, 52, 1667.

5. B. H. Westerink and T. I. Cremers, "Handbook of Microdialysis: Methods, Applications and Perspectives", 2007, Vol. 16, Academic Press.

6. T. E. Robinson and J. B. Justice, "Microdialysis in the Neurosciences: Techniques in the Behavioral and Neural Sciences", 2013, Vol. 7, Elsevier.

7. J. A. Stenken, E. M. Topp, M. Z. Southard, and C. E. Lunte, Anal. Chem., 1993, 65, 2324.

8. P. Nandi and S. M. Lunte, Anal. Chim. Acta, 2009, 651, 1.

9. S. Menacherry, W. Hubert, and J. B. Justice Jr., Anal. Chem., 1992, 64, 577.

10. M. I. Davies, J. D. Cooper, S. S. Desmond, C. E. Lunte, and S. M. Lunte, Adv. Drug Delivery Rev., 2000, 45, 169.

11. S. Y. Zhou, H. Zuo, J. F. Stobaugh, C. E. Lunte, and S. M. 
Lunte, Anal. Chem., 1995, 67, 594.

12. Z. D. Sandlin, M. Shou, J. G. Shackman, and R. T. Kennedy, Anal. Chem., 2005, 77, 7702.

13. P. Tủma, M. Šustková-Fišerová, F. Opekar, V. Pavlíček, and K. Málková, J. Chromatogr. A, 2013, 1303, 94.

14. M. Lorenzo, A. Navarrete, C. Balderas, and A. Garcia, J. Pharm. Biomed. Anal., 2013, 73, 116.

15. K. B. Male and J. H. Luong, J. Chromatogr. A, 2001, 926, 309.

16. X. Liu, L.-X. Yang, and Y.-T. Lu, J. Chromatogr. A, 2003, 998, 213.

17. P. Nandi, D. P. Desai, and S. M. Lunte, Electrophoresis, 2010, 31, 1414.

18. P. Nandi, D. E. Scott, D. Desai, and S. M. Lunte, Electrophoresis, 2013, 34, 895.

19. V. Poinsot, C. Bayle, and F. Couderc, Electrophoresis, 2003, 24, 4047.

20. R. A. Saylor and S. M. Lunte, J. Chromatogr. A, 2015, 1382, 48.

21. A. P. Mayer, I. Osorio, and C. E. Lunte, Epilepsy Behav., 2013, 29, 478.
22. W. K. T. Coltro, S. M. Lunte, and E. Carrilho, Electrophoresis, 2008, 29, 4928.

23. T. H. Linz, C. M. Snyder, and S. M. Lunte, J. Lab. Autom., 2012, 17, 24.

24. C. T. Culbertson, T. G. Mickleburgh, S. A. Stewart-James, K. A. Sellens, and M. Pressnall, Anal. Chem., 2013, 86, 95.

25. P. De Montigny, J. F. Stobaugh, R. S. Givens, R. G. Carlson, K. Srinivasachar, L. A. Sternson, and T. Higuchi, Anal. Chem., 1987, 59, 1096.

26. J. E. Melanson and C. A. Lucy, Analyst, 2000, 125, 1049.

27. M. F. Mora, A. M. Stockton, and P. A. Willis, Electrophoresis, 2012, 33, 2624.

28. C. T. Culbertson, Y. Tugnawat, A. R. Meyer, G. T. Roman, J. M. Ramsey, and S. R. Gonda, Anal. Chem., 2005, 77, 7933.

29. N. Adachi, Y. Itoh, R. Oishi, and K. Saeki, J. Cereb. Blood Flow Metab., 1992, 12, 477.

30. J. A. Alabadí, J.-L. Thibault, E. Pinard, J. Seylaz, and F. Lasbennes, Brain Res., 1999, 839, 305.

31. R. Böckelmann, M. Reiser, T. Horn, and G. Wolf, Amino Acids, 2000, 19, 423. 